\title{
Holographic interferometry study of the dissolution and diffusion of gypsum in water
}

\author{
JeAn Colomban* and Jacques BerT \\ Laboratoire de Physique de la Matière Condensée et Nanostructures, Université Claude Bernard Lyon 1; \\ CNRS, UMR 5586, Domaine scientifique de la Doua, F-69622 Villeurbanne cedex, France
}

\begin{abstract}
Revised version - 29 November 2006
Abstract - We have performed holographic interferometry measurements of the dissolution of the (010) plane of a cleaved gypsum single crystal in pure water. These experiments have provided the value of the dissolution rate constant $k$ of gypsum in water and the value of the interdiffusion coefficient $D$ of its aqueous species in water. $D$ is $1.0 \times 10^{-9} \mathrm{~m}^{2} \mathrm{~s}^{-1}$, a value close to the theoretical value generally used in dissolution studies. $k$ is $4 \times 10^{-5} \mathrm{~mol} \mathrm{~m}^{-2} \mathrm{~s}^{-1}$. It directly characterizes the microscopic transfer rate at the solid-liquid interface, and is not an averaged value deduced from quantities measured far from the surface as in macroscopic dissolution experiments. It is found to be two times lower than the value obtained from macroscopic experiments.
\end{abstract}

\section{INTRODUCTION}

Pressure Solution Creep (PSC) constitutes a major plastic strain process of immersed solids under low stress. A crystal wetted by a solvent (generally water) and saturated by its components is at chemical equilibrium. But if that crystal is then submitted to an external stress, it experiences dissolution. This originates in the chemical potential change of the solid induced by the stress. The dissolved species then diffuse away from the high stress region and precipitate in a stress-free zone. This mechanism is known to play a chief role in the upper crust and contributes, for instance, to upper crust deformation and to the diagenesis of sedimentary rocks.

The study of PSC in gypsum is of double interest. First it controls the upper crust strength in numerous geological situations because of its high ductility (deMeer and Spiers, 1997). Additionally, PSC in gypsum also likely plays a role in the high ductility and low strength of plaster, which is essentially made of gypsum needles, in the presence of moisture (Chappuis, 1999).

To understand the mechanisms involved in PSC in gypsum, precise values of the involved quantitative parameters are needed. Among them, the diffusion coefficient and the dissolution rate constant are primordial. Furthermore, these values are also of interest in other fields. Wide areas of gypsum karsts exist world-wide The instability of these karsts and potential collapse undermines the importance of understanding karst evolution and subsequently, the importance of knowing the precise values involved in the water-gypsum interaction (Jeschke et al., 2001). The presence of the dissolved species of gypsum in water influences the dissolution of minerals containing pollutants (especially in uranium mines). Hence the study of such contamination also necessitates values regarding gypsum dissolution (Kuechler et al., 2004). The existence of a large quantity of dissolved mineral may also alter the quality of the drinking water (Raines and Dewers, 1997). Lastly, knowledge of gypsum dissolution/precipitation behaviour is required in the oil and gas industry where gypsum is a common annoying "scale" mineral (Raju and Atkinson, 1990).

Surprisingly, no diffusion coefficient measurements of gypsum in water are available in the literature Lobo and Quaresma, 1989; Zavtsev and Asevev, 1992). Recent studies of gypsum dissolution either estimate the interdiffusion coefficient of the aqueous components (Raines and Dewers, 1997) or make use of a numerical value computed in 1971 (Barton and Wilde, 1971) with a Nernst-Hartley equation from the tracer diffusivity of the ions (Jeschke et al., 2001). Classical dissolution measurements typically use rotating-disk setups, batch-experiments or column-experiments with powder or crystal samples. In these apparatuses, the concentration during dissolution is monitored by techniques such as titrimetry, conductimetry, atomic absorption spectroscopy, mass spectrometry or colorimetry. During these experiments, water is usually flowing and the overall measured concentration stems from a combination of diffusion, forced convection and dissolution. If empirical equations always enable one to describe the measurements, the link with pure phenomena is not straightforward and needs accurate knowledge of the surface morphology and of the transport properties of the system to be reliable (Jeschke and Drevbrodt, 2002a). To overcome these experimental limitations, we have carried out local, instead of global, experiments of gypsum dissolution in water with an alternative technique, holographic interferometry. This method allows simultaneous determination of the dissolution rate constant of gypsum in water at the dissolving interface and of the diffusion coefficient of its components in bulk water. The fundamental difference between our measurements and standard ones lies in the fact

*Author to whom correspondence should be addressed (Jean.Colombani@lpmcn.univ-lyon1.fr). 
that we look directly at the surface behavior instead of deducing this behavior from quantities measured far from the surface.

\section{EXPERIMENTS}

Historically, real-time holographic interferometry has been recognized as a valuable tool in solution chemistry (Knox et al., 1967). One of its advantages is that defects along the optical path (except in the working cell) compensate between the two light expositions of the hologram (see below), thus requiring less demanding setups than classical interferometry devices (Colombani and Bert, 2006). Furthermore the observation of the two-dimensional concentration field in the liquid enables the identification of any non-diffusive fluxes (natural convection, gravitational instability, ...), thus providing a guarantee of the reliability of the measurements (Colombani et al., 1998 ).

Our setup is designed as follows (see Fig. 1). The beam of a Diode Pumped Solid State laser $(\lambda=532$ $\mathrm{nm}$ wavelength) is divided into a reference beam and an object beam by a $50 / 50$ beam splitter. The two beams cross a half-wave plate and a Glan-Taylor prism (vertically polarizing), which act as a polarizer/analyzer system controlling the intensity of the beam. The two beams are both expanded and spatially filtered by a microscope objective/pinhole/convergent lens set. A parallel plate installed on a rotating mount is inserted between the pinhole and the convergent lens of the reference beam. This device allows the removal of eventual parasitic fringes on the initial interferogram. The two beams interfere on the holographic plate, their polarization vectors being parallel and colinear to the plate. The entire setup is located on a vibration-damping structure.

Air motion in the laboratory causes a random change with time of the path length along the beams, creating parasitic vibrations of the fringes. To reduce this source of uncertainty, the entire optical table is covered with a closed shelter and the real-time optical data acquisition is performed in a contiguous room instead of in the laboratory.

The primary interest of holography lies in the fact that the interference pattern of the object and the reference beams contains the amplitude (as in classical photography) and the phase of the object beam. In this way, the amplitude and phase of the object at time $t_{0}$ are recorded on the hologram. Therefore, when both the object and the hologram (containing the memory of the object at time $t_{0}$ ) are enlightened at time $t$ by the laser, the phase difference $\Delta \phi$ of the object between times $t_{0}$ and $t$ are visualized through $N=\Delta \phi /(2 \pi)$ interference fringes (interferograms). The interferograms are visualized and recorded with a Charge-Coupled Device camera, a monitor and image acquisition software.

This device, thus, gives access to any evolution of the phase $\Delta \phi=2 \pi e \Delta n / \lambda$ ( $e$ path length in the optical cell) and consequently to any evolution of the refractive index $\Delta n$ of the liquid (all other optical components being kept unchanged). Therefore, the change of solute molality $\Delta m=\Delta n /(\partial n / \partial m)=N \lambda /(e(\partial n / \partial m))$ in the solution can be registered $(\partial n / \partial m$ is the derivative of the index of refraction $n$ of the solution with the solute molality $m)$.

The crystal/solution system is located in an optical cell inside a copper structure with circulating thermostated water. The temperature of the water bath is regulated by a PID regulator, which probes the temperature with a platinum thermistor inserted into the copper structure. Indeed a temperature variation may be the source of a solubility, diffusivity and dissolution rate constant change. Therefore, constant temperature must be guaranteed ( $\pm 0.01 \mathrm{~K}$ in our experiments).

An experiment proceeds as follows: The optical cell is filled with ultrapure water. The liquid is left in the copper structure for a few hours in order to obtain a homogeneous temperature in the system. Subsequently, a reference hologram is taken. Then a cleaved gypsum single crystal $\left(\mathrm{CaSO}_{4}, 2 \mathrm{H}_{2} \mathrm{O}\right)$ from the Mazan mine (Vaucluse, France) of rough dimensions $5 \times 5 \times 1 \mathrm{~mm}^{3}$ is introduced into the cell, this time being considered as the time origin of the reaction. Interferograms are then recorded periodically (see Fig. 21). The white homogeneous zone above the fringes in the first interferograms is considered to be pure water (the inhomogeneous grey zone is a parasitic fringe). Indeed, no change of color is observable in this part of the cell compared to the reference hologram, so the phase has remained unchanged and we may infer that no discernible gypsum has migrated yet to this zone. Proceeding downwards, an initial black fringe is encountered, revealing a change of $\pi$ in the phase of the transmitted beam, compared to the flat-colored zone just above. Hence, we can conclude that the solute molality has increased at this point until it has reached a value for which the resulting change of phase is $\pi$. This corresponds to a molality change $\Delta m=\lambda /(2 e(\partial n / \partial m))$ (cf. above). The liquid just above the fringe has been identified as pure water therefore the molality all along this fringe is merely $m=\Delta m$. The adjacent white fringe, immediately below, corresponds to a $\pi$ change of the phase compared to the black fringe, and a $2 \pi$ evolution compared to the flat-colored zone. The molality at this level is then $m=2 \Delta m$. The adjacent black fringe, just below, corresponds to a $\pi$ change of the

phase compared to the white fringe, and a $3 \pi$ evolution compared to the flat-colored zone. The molality at this level is then $m=3 \Delta m$. The molality in the whole cell is then gradually reconstructed (see Fig. 30). 
The limiting factor of the spatial resolution of the interferogram is the number of pixels in the camera sensor. The larger the pixel number, the smaller the area depicted by one pixel. The size of the chip of our camera is $752 \times 582$ pixels and our resolution $40 \mu \mathrm{m}$. This value is small enough to provide us with a correct discretization of the molality in the cell.

\section{DATA ANALYSIS}

The investigated chemical reaction can be written as :

$$
\mathrm{CaSO}_{4} \cdot 2 \mathrm{H}_{2} \mathrm{O}(\mathrm{s}) \leftrightharpoons \mathrm{Ca}^{2+}(\mathrm{aq})+\mathrm{SO}_{4}^{2-}(\mathrm{aq})+2 \mathrm{H}_{2} \mathrm{O}(\mathrm{l})
$$

It takes place at the lower end of our cell. Considering the symmetry of the experiment, it can be reduced to a one-dimensional diffusion problem along the vertical coordinate $z$. In the absence of other added salts, the two aqueous species are present at each $z$ value with the same molality to preserve local electroneutrality. Therefore, $m_{\mathrm{Ca}^{2+}}(z, t)=m_{\mathrm{SO}_{4}^{2-}}(z, t), m_{\mathrm{Ca}^{2+}}(z, t)$ and $m_{\mathrm{SO}_{4}^{2-}}(z, t)$ being the molalities of $\mathrm{Ca}^{2+}(\mathrm{aq})$ and $\mathrm{SO}_{4}^{2-}(\mathrm{aq})$, respectively, at position $z$ and time $t$. Our investigation method is solely sensitive to the change of refractive index $n$, regardless of the species causing this change. In fact, both ions induce the modification of $n$, plus a possible contribution of ion pairs and impurities. Therefore, we experimentally access an effective molality of dissolved gypsum $m(z, t) \approx$ $m_{\mathrm{Ca}^{2+}}(z, t)=m_{\mathrm{SO}_{4}^{2-}}(z, t)$.

To evaluate $m$ along the vertical coordinate as a function of time, Fick's second law is used. if the diffusion coefficient is considered as constant in our molality range (see Section 4), this law reads:

$$
\left(\frac{\partial m}{\partial t}\right)_{z}=D\left(\frac{\partial^{2} m}{\partial z^{2}}\right)_{t}
$$

with $D$ as the interdiffusion coefficient of the solution, and must be solved in space and time with the following boundary conditions (all the symbols used are summed up in Table 1):

- Mass balance at the dissolving interface implies the amount of gypsum leaving the solid and entering the liquid to being equal:

$$
F_{\text {dissolution }}(0, t)=F_{\text {diffusion }}(0, t)
$$

where $F$ is the flow rate and the origin of coordinate is the geometric solid-liquid interface, considered as fixed (actually moving of less than one pixel in the course of a typical experiment).

The expression of the dissolution flow rate is:

$$
F_{\text {dissolution }}(0, t)=\frac{d \xi}{d t}
$$

with $\xi$ the advancement variable (amount of transformed reagent). If we follow a transition states theory (Shiraki and Brantley, 1995), the rate of advancement $d \xi / d t$ takes the form:

$$
\frac{d \xi}{d t}=k s_{r} a_{s o l}\left[1-\exp \left(-\frac{\mathcal{N} A}{R T}\right)\right]
$$

where $k$ is the dissolution rate constant of gypsum in water, $s_{r}$ is the total dissolving gypsum-water interface, $a_{s o l}$ is the activity of the reagent (conventionally taken as one for a solid), $A$ is the chemical affinity of the reaction, $R$ is the gas constant, $T$ is the absolute temperature and $\mathcal{N}$ is a constant. The chemical affinity of dissolution, in other words the change of Gibbs free energy when gypsum changes its thermodynamic state from solid to dissolved, is $A=-R T \ln \Omega$. In this expression, $\Omega$ stands for the supersaturation:

$$
\Omega=\frac{a_{\mathrm{Ca}^{2}+} a_{\mathrm{SO}_{4}^{2-}} a_{\mathrm{H}_{2} \mathrm{O}}^{2}}{K_{s p}} .
$$

$a_{X}$ is the activity of the subscripted aqueous species and $K_{s p}$ is the solubility product of the reaction of Eq.

囯. The solubility product can be written as: $K_{s p}=a_{\mathrm{Ca}^{2+}}^{\mathrm{sat}} a_{\mathrm{SO}_{4}^{2-}}^{\mathrm{sat}}\left(a_{\mathrm{H}_{2} \mathrm{O}}^{\mathrm{sat}}\right)^{2}$, where $a_{X}^{\mathrm{sat}}$ represents the activity

\footnotetext{
†Our standard state is characterized by a unit activity for pure gypsum (solid reagent) and pure water (solvent) at any temperature and pressure. For the aqueous species of the solute, it is a unit activity in a $1 \mathrm{~mol} \mathrm{~kg}^{-1}$ solution representing an infinitely dilute solution, for any temperature and pressure.
} 
of the subscripted species at chemical equilibrium. As the solubility of gypsum is small, the activity of the solvent $a_{\mathrm{H}_{2} \mathrm{O}}$ is always considered to be equal to one. If we introduce the mean ionic activity coefficient $\gamma_{ \pm}$, geometric mean of the two single ion activity coefficients, the supersaturation can be rewritten:

$$
\Omega=\frac{\gamma_{ \pm}^{2} m_{\mathrm{Ca}^{2+}} m_{\mathrm{SO}_{4}^{2-}}}{\left(\gamma_{ \pm}^{\mathrm{sat}}\right)^{2} m_{\mathrm{Ca}^{2+}}^{\mathrm{sat}^{\mathrm{sat}}} m_{\mathrm{SO}_{4}^{2-}}}
$$

$m_{X}^{\text {sat }}$ is the molality of the aqueous component $X$ at chemical equilibrium, and $\gamma_{ \pm}^{\text {sat }}$ is the mean ionic activity coefficient at chemical equilibrium. Here we make the reasonable assumption that at all the ionic molalities achieved in our experiments, the ionic strength remains small enough so that the mean ionic activity coefficient does not depart from its value in pure water. This term can thus be suppressed from the expression of Eq. 7

In the absence of experimental or theoretical determination of the value of the constant $\mathcal{N}$ in Eq. 5 , we have used the value successfully used for fitting calcite dissolution measurements (Rickard and Siöberg, 1983; Shiraki and Brantley, 1995): $\mathcal{N}=1 / 2$. This choice results in the dissolution flow rate at the solid-liquid interface:

$$
F_{\text {dissolution }}(0, t)=k s_{r}\left(1-\frac{\sqrt{m_{\mathrm{Ca}^{2+}}(0, t) m_{\mathrm{SO}_{4}^{2-}}(0, t)}}{\sqrt{m_{\mathrm{Ca}^{2+}}^{\mathrm{sat}} m_{\mathrm{SO}_{4}^{2-}}^{\mathrm{sat}}}}\right) .
$$

We will identify the geometric mean of the ionic molalities with our effective molality $m$. The geometric mean of the ionic solubilities is well known and will be written $m^{\text {sat }}$. Finally, the dissolution flow rate at the surface reduces to:

$$
F_{\text {dissolution }}(0, t)=k s_{r}\left(1-\frac{m(0, t)}{m^{\text {sat }}}\right) .
$$

The diffusion flow rate is linked to the diffusion flux at the dissolving interface $J_{\text {diffusion }}(0, t)$ merely through:

$$
J_{\text {diffusion }}(0, t)=\frac{1}{s} F_{\text {diffusion }}(0, t)
$$

where $s$ is the cell section perpendicular to the mass transport. This flux derives readily from Fick's first law:

$$
J_{\text {diffusion }}(0, t)=-D \rho\left(\frac{\partial m(0, t)}{\partial z}\right)_{t}
$$

with $\rho$ as the density of the solution. The introduction of Eq. 910 and 11 into Eq. 3induces for the boundary condition at the lower end of the cell:

$$
\left(\frac{\partial m(0, t)}{\partial z}\right)_{t}=-\frac{k \beta}{D \rho}\left(1-\frac{m(0, t)}{m^{\mathrm{sat}}}\right)
$$

where we have introduced $\beta=s_{r} / s$.

- At the upper end of the cell (water-air meniscus), the boundary condition is merely written $J_{\text {diffusion }}(L, t)=0$, $L$ being the height of water in the cell.

To get a tractable analytical expression of $m(z, t)$, we make a further assumption concerning the geometry of our experiment. Our cell is high enough and the investigated time short enough to consider that gypsum diffuses in a semi-infinite medium. This hypothesis has been experimentally verified, paying attention to the fact that the highest molality fringe has not reached the top of the cell at the end of the measurement. The solution of Equation 2 is in this case (Crank, 1975):

$$
m(z, t)=m^{\mathrm{sat}}\left[\operatorname{erfc}\left(\frac{z}{2 \sqrt{D t}}\right)-\exp \left[\frac{k \beta z}{D \rho m^{\mathrm{sat}}}+\left(\frac{k \beta}{D \rho m^{\mathrm{sat}}}\right)^{2} D t\right] \times \operatorname{erfc}\left[\frac{z}{2 \sqrt{D t}}+\frac{k \beta}{D \rho m^{\mathrm{sat}}} \sqrt{D t}\right]\right] .
$$

erfc is the complementary error function. The values of $m^{\text {sat }}, D$ and $k$ are obtained through a fit of our experimental $m(z, t)$ curves with the above expression. The shift to the right of the molality curves (Fig. 3) can be viewed as a signature of diffusion, and the shift of the molality curves upward can be viewed as a signature of dissolution. 


\section{RESULTS}

$\partial n / \partial m$ is a value of primary importance, but unfortunately no experimental determination is available. Therefore, we have carried out refractive index measurements with an Abbe refractometer in pure water and water where gypsum was dissolved until saturation at ambient temperature $\left(m^{\text {sat }}=15 \mathrm{mmol} \mathrm{kg}{ }^{-1}\right.$ corresponding to $2 \mathrm{~g} \mathrm{l}^{-1}$ (Raju and Atkinson, 1990)). These experiments were not far from the resolution limit of the apparatus. A (0.019 $\pm 0.007) \mathrm{kg} \mathrm{mol}^{-1}$ value was found, which was used for the refractive index into molality conversion.

To guarantee the validity of the data analysis, Equation 2 requires $D$ to remain constant with molality. Unfortunately no experimental determination of $D$ exists, and furthermore of the dependance of $D$ on molality. To obtain an initial, crude idea of the variation $\Delta D$ of the diffusion coefficient of gypsum in water in the molality range $\Delta m$ between pure water and saturated solution $\left(\Delta m=m^{\text {sat }}=15 \mathrm{mmol} \mathrm{kg} \mathrm{k}^{-1}\right)$, we have collected the $\partial D / \partial m$ values of other calcium and sulfate salts in water (Lobo and Quaresma, 1989; Zaytsev and Asevev, 1992) and computed their $\Delta D$ for the same molality range. The worst case is $\mathrm{ZnSO}_{4}$ at low molality with $\Delta D / D \sim 20 \%$. Evidently, no strict conclusion can be inferred for gypsum from that value, but a relatively weak dispersion of $D$ in our experimental cell can be expected.

Precipitation and dissolution of minerals are known to be strongly influenced by the presence of impurities. To evaluate the purity of our single crystals, we carried out the Electron Probe Microanalysis of a sample of our material. No elements, other than the atoms constituting gypsum, have been detected with a concentration greater than $0.1 \%$. The known impurities found in the gypsum of the Mazan mines, possibly present with a lower concentration, are dolomite rocks, quartz, anhydrite and celestite.

For the computation of $\beta=s_{r} / s$ used in Equation 13. the cross-diffusional section area $s$ is taken as the horizontal section of our optical cell $\left(s=0.95 \mathrm{~cm}^{2}\right)$ and $s_{r}$ is taken as the dissolving surface area of the single crystal $\left(s_{r} \approx 0.3 \mathrm{~cm}^{2}\right)$.

We have measured the $p H$ of our solution at the end of the experiment and observe a $\mathrm{pH}$ of 5.7, which roughly corresponds to the $p H$ of the $\mathrm{CO}_{2} / \mathrm{HCO}_{3}^{-}$system stemming from the dissolution of the atmospheric $\mathrm{CO}_{2}$ in water.

Seven measurements, all at $20.00^{\circ} \mathrm{C}$, have been performed to check the reproducibility. The fit of the experimental points by the theoretical law of Eq. 13 brings a solubility $m^{\text {sat }}=15.6 \mathrm{mmol} \mathrm{kg}^{-1}$, which is very close to the expected value of $15 \mathrm{mmol} \mathrm{kg} \mathrm{kg}^{-1}$ (Raju and Atkinson, 1990). This agreement validates our analysis procedure and particularly the chosen $\partial n / \partial m$ value. We find a diffusion coefficient $D=(1.0 \pm 0.1) \times 10^{-9} \mathrm{~m}^{2} \mathrm{~s}^{-1}$, a value very close to the $0.9 \times 10^{-9} \mathrm{~m}^{2} \mathrm{~s}^{-1}$ value derived from Nernst-Hartley equation, generally used in dissolution studies (Barton and Wilde, 1971). We find a dissolution rate constant $k=(4 \pm 1) \times 10^{-5} \mathrm{~mol} \mathrm{~m}^{-2} \mathrm{~s}^{-1}$. The statistical uncertainty is the standard error, computed from the seven measurements.

\section{MIXED KINETICS}

There has been a strong debate to clearly determine the nature and the kinetics of the slowest step of the dissolution process, controlling the kinetics. If mass transport in the liquid (proportional to the diffusion coefficient $D$ ) is slow compared to the chemical reaction at the solid surface (proportional to the dissolution rate constant $k$ ), ions are rapidly unbound from the solid but slowly transported away in the solution, hence the dissolution global kinetics is controlled by molecular diffusion. Conversely, if mass transport proceeds quickly compared to the reaction rate, the kinetics is controlled by the slow ion detachment from the mineral. Both macroscopic studies - rotatingdisk, column-experiments ... Raines and Dewers, 1997; Drevbrodt and Gabrovsek, 2000; Dewers and Raines, 2000; Jeschke et al., 2001) - and microscopic studies - atomic force microscopy (Bosbach and Rammensee, 1994; Hall and Cullen, 1996) - have tried to address the question.

One of the reasons of the diversity of the available results lies in the fact that the balance between transport and reaction strongly depends on the geometry of the system and on the thermodynamic conditions. Jeschke et al. (2001) conclude with mixed kinetics, where both effects are of comparable magnitude, with the linear kinetics of Eq. $9\left(F_{\text {dissolution }} \sim m\right.$, except very close to the saturation $)$ for the dissolution. This conclusion is drawn from a combination of batch dissolution and rotating-disk experiments. Therefore, the authors make use of the formalism of these kinds of methods to evaluate the two phenomena: the dissolution velocity is evaluated by $k_{s}$, an empirical dissolution rate constant, and the diffusion velocity is evaluated by $k_{t}=D c_{\mathrm{eq}} / \varepsilon$, a transport constant $\left(c_{\mathrm{eq}}\right.$ stands for the molarity of aqueous calcium at saturation and $\varepsilon$ for the thickness of the diffusion boundary layer surrounding the crystal). The authors find $k_{t}=1.5 \times 10^{-3} \mathrm{~mol} \mathrm{~m}^{-2} \mathrm{~s}^{-1}$ from batch experiments and $k_{s}=1.3 \times 10^{-3} \mathrm{~mol}$ $\mathrm{m}^{-2} \mathrm{~s}^{-1}$ from both experiments (for $c / c_{\mathrm{eq}}<0.94$ ). These two values are so close that the mixed aspect of the dissolution kinetics is unambiguously assessed for this situation. Beyond the interest of this result, one can see that the selected evaluation quantities are strongly linked to the experimental methods. 
Besides, Murphy et al. (1989) have proposed the ratio of the maximum diffusive and reactive flow rates $\alpha=$ $D \rho m^{\text {sat }} /(\beta k L)$ to be a dimensionless number used to discriminate between transport-controlled and reactioncontrolled dissolution. This number has a more universal impact, with no quantity intrinsic to the chosen experiments (like $\varepsilon$ above). Pure transport control corresponds theoretically to $\alpha=0$, pure surface control to $\alpha=\infty$ and mixed control to $\alpha=1$. These authors have numerically investigated realistic geological dissolution configurations of quartz and calcite in water in geometries comparable to ours (zero-flux boundary) and conclude that at values of $\alpha$ between $10^{-2}$ and 10 , the rate of evolution of the system is controlled by mixed surface reaction and diffusion kinetics.

Our experimental values lead, with $L=3 \mathrm{~cm}$ and $\rho=10^{3} \mathrm{~kg} \mathrm{~m}^{-3}$, to $\alpha=0.05$. This reveals mixed kinetics, according to the classification of Murphy et al. At ambient temperature and pressure, the mixed kinetics of dissolution of gypsum in water seems to be a robust feature, in our geometry (a reacting surface at one boundary and a zero flux at the other) as well as in the geometry of Jeschke et al. (2001) (a reacting surface at one boundary and a constant composition reservoir at the other).

\section{COMPARISON BETWEEN LOCAL AND GLOBAL DISSOLU- TION MEASUREMENTS}

The comparison of our dissolution rate constant $k$ values with results of global measurements requires particular care. As has been often stated (Rickard and Siöbero, 1983; Jeschke and Drevbrodt, 2002b), derivation of rate constants from these kinds of experiments is a difficult task and the procedure strongly depends on the device geometry and sample morphology.

To enter into the details of a global experiment, the overall flux $\mathcal{R}$ in a vessel of volume $v$ is computed from the time evolution of the overall concentration $c$ and is considered to be identically equal to the diffusion and dissolution fluxes: $\mathcal{R}=J_{\text {dissolution }}=J_{\text {diffusion }}=(v / s)(d c / d t)$. But as water is flowing in the vessel, this equality is only valid at the frontier between the diffusional boundary layer and the bulk liquid, in other words along the section called $s$ above. Indeed this section corresponds to the geometrical locus where mass balance of Eq. 3 applies. This statement remains true exclusively for mixed kinetics. For pure reaction-controlled dissolution, mass balance applies at the solid-liquid interface, called $s_{r}$ above, and for pure transport-controlled dissolution, no information on dissolution coefficients can be obtained (Jeschke and Drevbrodt, 2002a).

Between the solid surface and the extremity of the boundary layer, there is no loss of solute, therefore mass balance imposes the flow rates at these two surfaces to equalize. At the solid-liquid interface (of area $s_{r}$ ), the flow rate $F_{r}$ can be deduced from an analog to Eq. 9. $F_{r}=s_{r} k\left(1-c / c_{\mathrm{eq}}\right)$. At the top of the diffusional boundary layer (of area $s$ ), the flow rate can be written $F_{d}=s k_{s}\left(1-c / c_{\text {eq }}\right)$ where $k_{s}$ is the empirical dissolution rate constant introduced in Section 5 (Jeschke et al., 2001). Accordingly $F_{r}=F_{d}$ implies $k=s k_{s} / s_{r}$ and we are now able to compare our microscopic dissolution rate constant $k$ with a rate constant $k_{s}$ deduced from a global dissolution measurement.

Recently, Jeschke et al. (2001) found a linear dissolution kinetics and $k_{s}=1.3 \times 10^{-3} \mathrm{~mol} \mathrm{~m}^{-2} \mathrm{~s}^{-1}$, as previously mentionned. The authors estimate the geometric specific surface area of their powders by optical microscopy to range between 60 and $73 \mathrm{~cm}^{2} \mathrm{~g}^{-1}$, depending on the gypsum type. We consider this surface to be similar to our diffusional section $s$. The BET-surface of these powders is $1100 \mathrm{~cm}^{2} \mathrm{~g}^{-1}$. We identify this surface with our dissolving interface $s_{r}$ 国. Therefore, one finds a $s / s_{r} \sim 1 / 15$ ratio, which gives a $k=9 \times 10^{-5} \mathrm{~mol} \mathrm{~m}^{-2} \mathrm{~s}^{-1}$ value.

Beside the uncertainty on the surface ratio, a factor of 2 or 3 between their global measurements $\left(9 \times 10^{-5} \mathrm{~mol}\right.$ $\left.\mathrm{m}^{-2} \mathrm{~s}^{-1}\right)$ and our local measurements $\left(4 \times 10^{-5} \mathrm{~mol} \mathrm{~m}^{-2} \mathrm{~s}^{-1}\right)$ seems to exist at first sight. But the large differences between the two methodologies could account for this discrepancy and we can consider these two values as being in fair agreement. One should mention that the $k$ we have measured concerns exclusively the dissolution of the (010) plane, whereas the $k_{s}$ of global experiments is an average of the dissolution rate constants of all the reacting planes of the dissolving powder. Therefore, the two values are not strictly comparable.

As a summary, microscopic measurements make the data interpretation easier for three reasons. First, there is no uncertainty on the surface where mass balance must be applied. Second, there is no flow in the cell and therefore no hydrodynamical assumptions and computations are needed. And finally the study of a single crystallographic interface avoid to obtain a multifaceted average dissolution rate constant.

\footnotetext{
$\ddagger$ Obviously this is questionable because the adsorption sites in the BET method and the dissolving sites in the dissolution experiment may slightly differ. But this assumption should at least bring the correct order of magnitude.
} 


\section{CONCLUSION}

We have used a non-invasive interferometric method to access to the microscopic dissolution rate constant of a cleaved (010) surface of gypsum in water. This constant has been revealed to be two times lower than the same constant measured by macroscopic averaged methods, which can be considered to be in fair agreement. The interdiffusion coefficient of the aqueous species of gypsum in water has also been measured during these experiments and exhibits a value close to the theoretical value generally used for dissolution studies. Now, to deepen the comprehension of pressure solution creep, we plan to perform the same experiments with gypsum crystals under stress. The knowledge of the influence of an uniaxial pressure on the dissolution parameters may shed new light on the mechanisms of PSC. 
Acknowledgements - We would like to thank Elisabeth Charlaix and Pierre Monchoux for fruitful discussions, Sylvain Meille (LCR Lafarge) for gypsum samples, Xavier Jaurand for the chemical analysis of gypsum, and Christophe Bineau, Alexandre Enderlin, Hervé Féret and Agnès Piednoir for experimental help. We are also very grateful to the anonymous referees and to the associate editor, Eric H. Oelkers, for their comments. Part of this study was funded by CNES (French spatial agency). 


\section{References}

Barton, A. and Wilde, N. (1971). Dissolution rates of polycristalline samples of gypsum and orthorhombic forms of calcium sulphate by a rotating disc method. Trans. Faraday Soc., 67, 3590.

Bosbach, D. and Rammensee, W. (1994). In situ investigation of growth and dissolution on the (010) surface of gypsum by Scanning Force Microscopy. Geochim. Cosmochim. Acta, 58, 843.

Chappuis, J. (1999). A model for a better understanding of the cohesion of hardened hydraulic materials. Colloids and surface, 156, 223.

Colombani, J. and Bert, J. (2006). Holographic interferometry for the study of liquids. J. Mol. Liq., in press.

Colombani, J., Dez, H., Bert, J., and Dupuy-Philon, J. (1998). Hydrodynamic instabilities and Soret effect in an aqueous electrolyte. Phys. Rev. E, 58, 3202 .

Crank, J. (1975). The mathematics of diffusion. Oxford University Press, Oxford.

deMeer, S. and Spiers, C. (1997). Uniaxial compaction creep of wet gypsum aggregates. J. Geophys. Res., 102, 875 .

Dewers, T. and Raines, M. (2000). Reply to comment on: mixed transport / reaction control of gypsum dissolution kinetics. Chem. Geol., 168, 275.

Dreybrodt, W. and Gabrovsek, F. (2000). Comments on: Mixed transport / reaction control of gypsum dissolution kinetics in aqueous solutions and initiation of gypsum karst by Michael A. Raines and Thomas A. Dewers in Chemical Geology 140, 29-48, 1997. Chem. Geol., 168, 169.

Hall, C. and Cullen, D. (1996). Scanning Force Microscopy of gypsum dissolution and crystal growth. AIChE, 42, 232.

Jeschke, A. and Dreybrodt, W. (2002a). Dissolution rates of minerals and their relation to surface morphology. Geochim. Cosmochim. Acta, 66, 3055.

Jeschke, A. and Dreybrodt, W. (2002b). Pitfalls in the determination of empirical dissolution rate equations of mineral from experimental data and a way out: an iterative procedure to find valid rate equations, applied to Ca-carbonates and -sulfates. Chem. Geol., 192, 183.

Jeschke, A., Vosbeck, K., and Dreybrodt, W. (2001). Surface controlled dissolution rates of gypsum in aqueous solutions exhibit nonlinear dissolution kinetics. Geochim. Cosmochim. Acta, 65, 27.

Knox, C., Sayano, R., Seo, E., and Silverman, H. (1967). Holographic interferometry in electrochemical studies. J. Phys. Chem., 71, 3102.

Kuechler, R., Noack, K., and Zorn, T. (2004). Investigation of gypsum dissolution under saturated and unsaturated water conditions. Ecological Modelling, 176, 1.

Lobo, V. and Quaresma, J. (1989). Physical sciences data 41, Handbook of electrolyte solutions, part B. Elsevier.

Murphy, W., Oelkers, E., and Lichtner, P. (1989). Surface reaction versus diffusion control of mineral dissolution and growth rates in geochemical processes. Chem. Geol., 78, 357.

Raines, M. and Dewers, T. (1997). Mixed transport / reaction control of gypsum dissolution kinetics in aqueous solutions and initiation of gypsum karst. Chem. Geol., 140, 29.

Raju, K. and Atkinson, G. (1990). The thermodynamics of "scale" mineral solubilities. 3. Calcium sulfate in aqueous NaCl. J. Chem. Eng. Data, 35, 361.

Rickard, D. and Sjöberg, E. (1983). Mixed kinetic control of calcite dissolution rates. Am. J. Sci., 283, 815.

Shiraki, R. and Brantley, S. (1995). Kinetics of near-equilibrium calcite precipitation at $100^{\circ} \mathrm{C}$ : An evaluation of elementary reaction-based and affinity-based rate laws. Geochim. Cosmochim. Acta, 59, 1457.

Zaytsev, I. and Aseyev, G. (1992). Properties of aqueous solutions of electrolytes. CRC Press, Boca Ratton. 


\section{List of Tables}

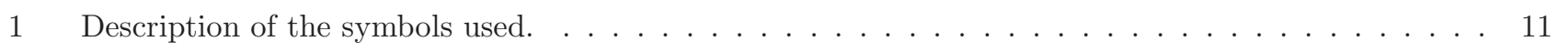




\begin{tabular}{|c|c|c|}
\hline Symbol & quantity & units \\
\hline$\beta$ & geometrical constant $\left(s_{r} / s\right)$ & \\
\hline$\gamma_{ \pm}$ & mean ionic activity coefficient & \\
\hline$\gamma_{ \pm}^{\text {sat }}$ & mean ionic activity coefficient at equilibrium & \\
\hline$\Delta \phi$ & phase difference & \\
\hline$\Delta D$ & diffusion coefficient difference & $m^{2} s^{-1}$ \\
\hline$\Delta m$ & molality difference & mol $\mathrm{kg}^{-3}$ \\
\hline$\Delta n$ & refractive index difference & \\
\hline$\varepsilon$ & thickness of a diffusion boundary layer & $\mathrm{m}$ \\
\hline$\lambda$ & laser wavelength & $\mathrm{m}$ \\
\hline$\xi$ & advancement variable & mol \\
\hline$\rho$ & density of the solution & $\mathrm{kg} \mathrm{m}^{-3}$ \\
\hline$\Omega$ & supersaturation & \\
\hline$A$ & chemical affinity of dissolution & $\mathrm{J} \mathrm{mol}^{-1}$ \\
\hline$a_{\mathrm{X}}$ & activity of species $X$ & \\
\hline$a_{\mathrm{X}}^{\mathrm{sat}}$ & activity of species $\mathrm{X}$ at equilibrium & \\
\hline$c$ & molarity of aqueous calcium & $\mathrm{mol} \mathrm{m}^{-3}$ \\
\hline$c_{e q}$ & molarity of aqueous calcium at equilibrium & $\mathrm{mol} \mathrm{m}$ \\
\hline$D$ & interdiffusion coefficient of the solution & $\mathrm{m}^{2} \mathrm{~s}^{-1}$ \\
\hline$e$ & path length in the optical cell & $\mathrm{m}$ \\
\hline$F$ & flow rate & $\mathrm{mol} \mathrm{s}^{-1}$ \\
\hline$J$ & flux & $\mathrm{mol} \mathrm{m}{ }^{-2} \mathrm{~s}^{-1}$ \\
\hline$k$ & dissolution rate constant & $\mathrm{mol} \mathrm{m}{ }^{-2} \mathrm{~s}^{-1}$ \\
\hline$k_{s}$ & empirical dissolution rate constant & $\mathrm{mol} \mathrm{m}{ }^{-2} \mathrm{~s}^{-1}$ \\
\hline$k_{t}$ & transport constant $\left(=D c_{e q} / \varepsilon\right)$ & $\mathrm{mol} \mathrm{m}{ }^{-2} \mathrm{~s}^{-1}$ \\
\hline$K_{s p}$ & solubility product & \\
\hline$L$ & height of water & $\mathrm{m}$ \\
\hline$m$ & effective molality of dissolved gypsum & $\mathrm{mol} \mathrm{kg}^{-1}$ \\
\hline$m^{\text {sat }}$ & effective molality of dissolved gypsum at equilibrium & mol kg-1 \\
\hline$m_{\mathrm{X}}$ & molality of species X & $\mathrm{mol} \mathrm{kg}{ }^{-1}$ \\
\hline$m_{\mathrm{X}}^{\text {sat }}$ & molality of species $\mathrm{X}$ at equilibrium & $\mathrm{mol} \mathrm{kg}{ }^{-1}$ \\
\hline$n$ & index of refraction & \\
\hline$N$ & number of interference fringes & \\
\hline $\mathcal{N}$ & numerical constant & \\
\hline$R$ & gas constant & $\mathrm{J} \mathrm{mol}^{-1} \mathrm{~K}^{-1}$ \\
\hline $\mathcal{R}$ & overall flux & $\mathrm{mol} \mathrm{m} \mathrm{m}^{-2} \mathrm{~s}^{-1}$ \\
\hline$s$ & area of the section perpendicular to mass transport & $\mathrm{m}^{2}$ \\
\hline$s_{r}$ & area of the dissolving interface & $\mathrm{m}^{2}$ \\
\hline$T$ & absolute temperature & $\mathrm{K}$ \\
\hline$t$ & time & $\mathrm{s}$ \\
\hline$z$ & vertical coordinate & $\mathrm{m}$ \\
\hline
\end{tabular}

Table 1: 


\section{List of Figures}

1 Holographic setup: DPSS laser, beam splitter, Glan-Taylor prism, half-wave plate with adjustable polarization direction, beam expander comprising a microscope objective, a pinhole and a converging lens, thermostated optical cell, holographic plate, mirrors, parallel plate on a rotating mount, camera. 13

2 Interferograms during the dissolution of a gypsum single crystal in pure water 9, 60, 120, 180 and 400 minutes after the beginning of the experiment. A black bar indicates the position of the crystal in one of the interferograms. . . . . . . . . . . . . . . . . . . . . 14

3 Evolution of the molality with vertical position during the experiment of Fig. 2, 9, 20, 30, 60, 75, 105, 120, 180, 240 and $360 \mathrm{~min}$. after the beginning of the experiment. Circles are for experimental points and lines for theoretical fit. The horizontal line is the solubility. . . . . . . . . . . . . 


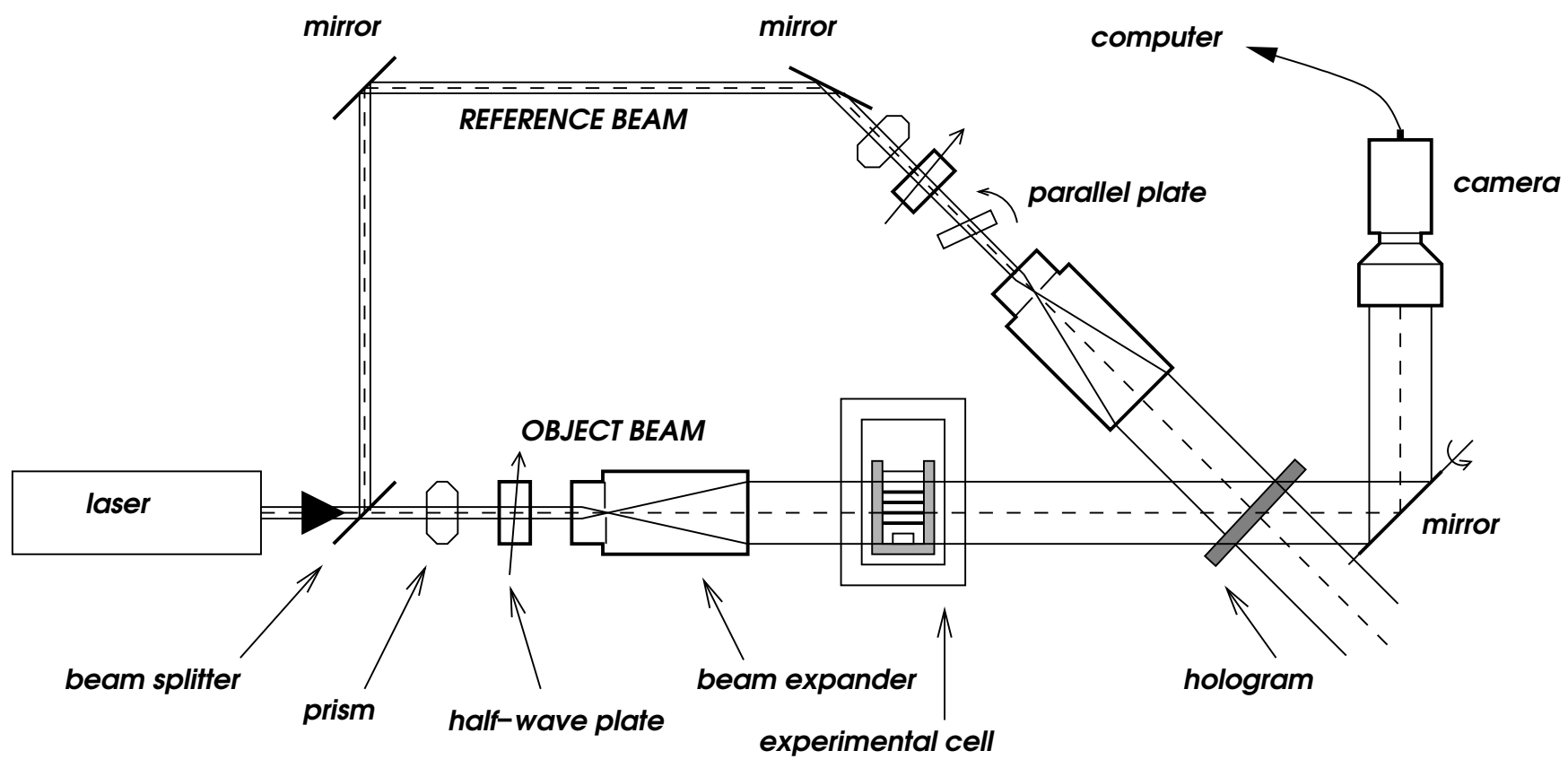

Figure 1: 


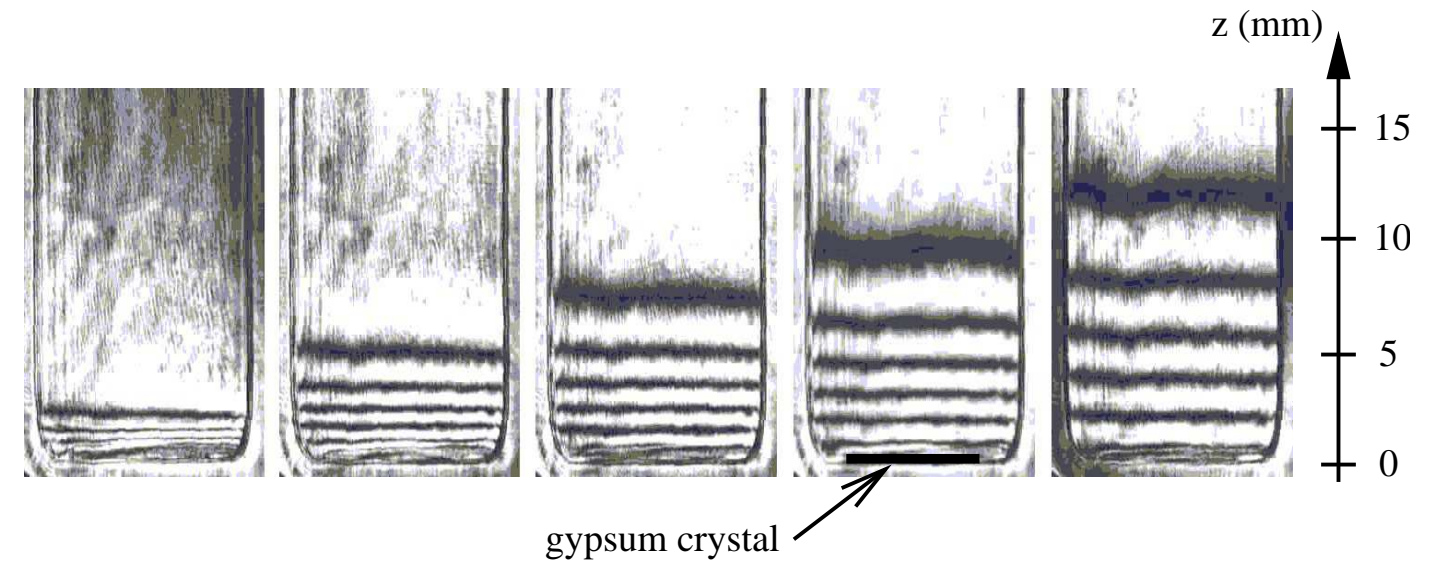

Figure 2: 


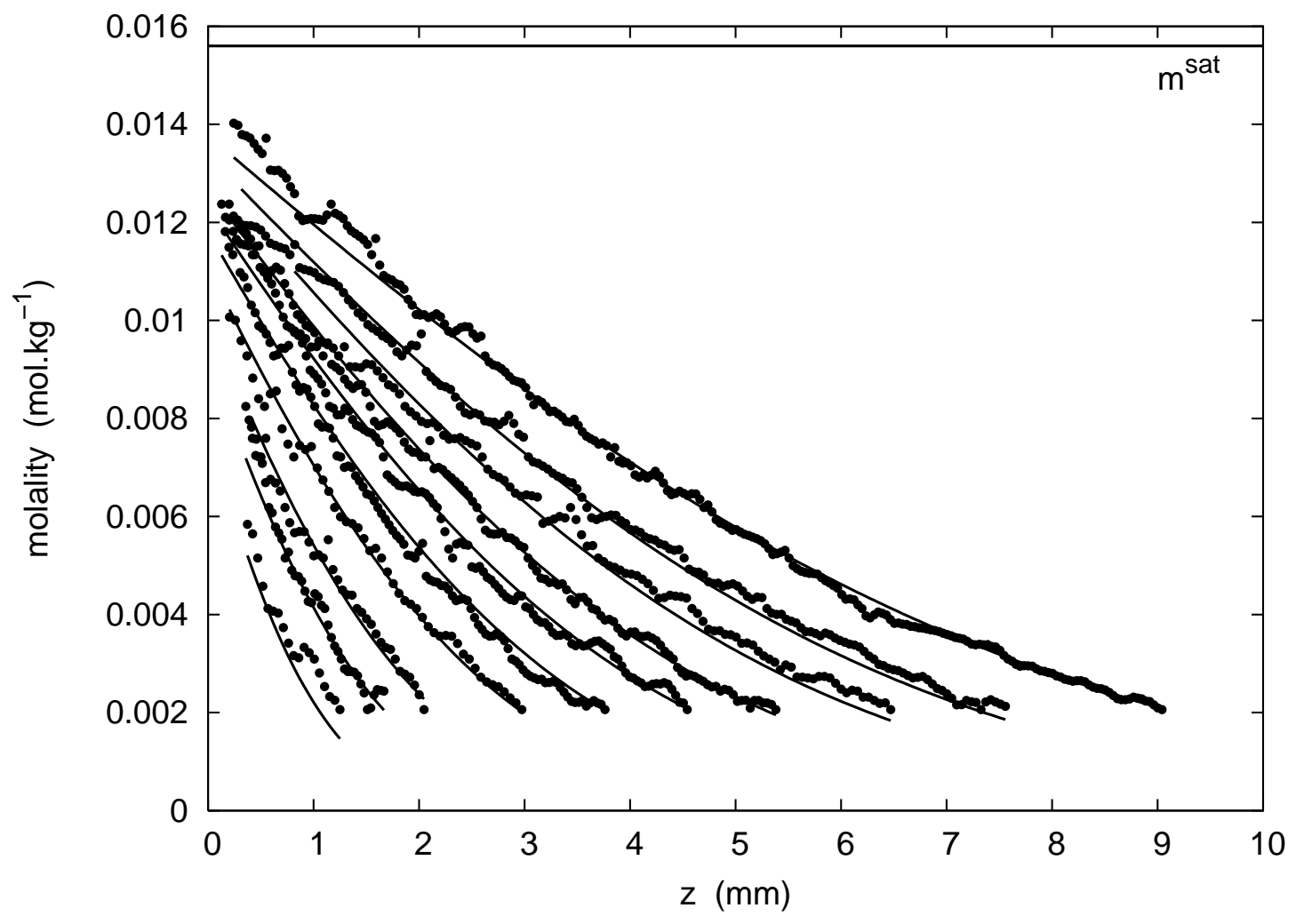

Figure 3: 